\title{
dspace.vutbr.cz
}

\section{Balanced-Output CCCFOA and Its Utilization in Grounded Inductance Simulator with Various Orders}

\author{
HERENCSÁR, N.
}

Proceedings of the 2018 41st International Conference on Telecommunications and Signal Processing (TSP)

pp. 188-191

elSBN: 978-1-5386-4695-3

DOI: http://dx.doi.org/10.1109/TSP.2018.8441349

Accepted manuscript

(C)2018 IEEE. Personal use of this material is permitted. Permission from IEEE must be obtained for all other uses, in any current or future media, including reprinting/republishing this material for advertising or promotional purposes, creating new collective works, for resale or redistribution to servers or lists, or reuse of any copyrighted component of this work in other works. Norbert Herencsar, "Balanced-Output CCCFOA and Its Utilization in Grounded Inductance Simulator with Various Orders", Proceedings of the 2018 41st International Conference on Telecommunications and Signal Processing (TSP), pp. 1-5, 2018. D0I: 10.1109/TSP.2018.8441349. Final version is available at https://ieeexplore.ieee.org/document/8441349/ 


\title{
Balanced-Output CCCFOA and Its Utilization in Grounded Inductance Simulator with Various Orders
}

\author{
Norbert Herencsar \\ Department of Telecommunications \\ Brno University of Technology \\ Technicka 3082/12, 61600 Brno, Czech Republic \\ Email: herencsn@feec.vutbr.cz
}

\begin{abstract}
In this paper, a new realization of currentcontrolled current feedback operational amplifier with balanced voltage outputs (BO-CCCFOA) is presented. A resistorless grounded lossless positive inductance simulator (PIS) using two BO-CCCFOAs and a grounded capacitor is reported. The resulting equivalent inductance value of PIS can be adjusted either via change of input intrinsic resistance of BO-CCCFOAs by means of biasing currents or by order of fractional-order capacitor $(\mathrm{FoC})$. FoCs of order $\gamma=(0.25 ; 0.5 ; 0.75 ; 1)$ were emulated via $5^{\text {th }}$-order Foster II RC network and values optimized using modified least squares quadratic (MLSQ) method. In frequency range $30 \mathrm{kHz}-30 \mathrm{MHz}$ the obtained phase angle deviation of FoCs and mean values of corresponding relative phase error are below \pm 1 degree and $\pm 4.3 \%$, respectively. Considering the bandwidth for phase angle deviation less than 3 degree, the proposed fractional-order PIS operates over two decades. The behavior of the PIS circuit with various orders was tested via implementation in $R L_{\gamma} C$ ladder prototype of voltagemode high-pass filter. Theoretical results are verified by SPICE simulations using TSMC $0.18 \mu \mathrm{m}$ level-7 LO EPI SCN018 CMOS process parameters with $\pm 1 \mathrm{~V}$ supply voltages.
\end{abstract}

Keywords-BO-CCCFOA; current-controlled current feedback operational amplifier; fractional-order capacitor; FoC; fractional-order inductor; FoI; positive inductance simulator; $\mathbf{R L}_{\gamma} \mathbf{C}$ prototype; voltage-mode.

\section{INTRODUCTION}

During the last decades, active circuits emulating passive inductor characteristics have received considerable attention. It is due to that fact that monolithic spiral inductors suffer from substrate resistive losses and capacitive couplings, while their active counterparts can be electronically tunable with relatively broad spread of inductance value and less costly [1]. Hence, designers' attention was focused on active inductance simulator design using various high performance analog building blocks (ABBs). Among the ABBs that can operate in both current and voltage mode, the current feedback operational amplifier (CFOA) is one of the most commonly used due to high slew rate, wide bandwidth, etc. [2]-[4]. This paper aims to increase the variety of inductance simulator circuits reported in the literature with a new resistorless grounded lossless positive inductance simulator (PIS) with various orders. Note that, currently the number of applications of fractional-order systems rapidly grows due to the fractional origin of the real-

This article is based upon work from COST Action CA15225, a network supported by COST (European Cooperation in Science and Technology). Research described in this paper was financed by the National Sustainability Program under grant LO1401 and by the Czech Science Foundation under grant no. 16-11460Y. For the research, infrastructure of the SIX Center was used. world phenomena [5]-[12]. In these systems the so-called fractional-order capacitors (FoCs) [5]-[8] or fractional-order inductors (FoIs) [8]-[12] are considered as the most crucial design components with an impedance expressed in the frequency domain as $Z(s)=D s^{-\gamma}$. Here, $\gamma$ is the order of the fractional-order component, which is known as the dispersion coefficient, and $D$ is for $0<\gamma<1$ the coefficient of pseudocapacitance of FoC expressed in units of Farad $\cdot \mathrm{Sec}^{\gamma-1}$. On the other hand, if $-1<\gamma<0, D$ expresses the coefficient of pseudoinductance of $\mathrm{FoI}$ in units of Henry $\cdot \mathrm{sec}^{\gamma-1}$. In this paper, first of all, three FoCs of order $\gamma=(0.25 ; 0.5 ; 0.75)$ emulated via $5^{\text {th }}$ order Foster II RC network and component values optimized for constant phase in broad frequency range are realized due to lack of commercially available FoCs. Subsequently, the workability of FoI employing two BO-CCCFOAs and designed FoCs were demonstrated in voltage-mode (VM) high-pass filter (HPF) $\mathrm{RL}_{\gamma} \mathrm{C}$ ladder prototype. The behavior of proposed circuits were verified by SPICE simulations.

\section{CIRCUIT DESCRIPTION}

The circuit symbol of the BO-CCCFOA is shown in Fig. 1(a). This ABB with capability of intrinsic input resistance electronic control and balanced voltage outputs is equivalent to a positive-type current-controlled second-generation current conveyor $(\mathrm{CCCII}+)$ with a differential voltage buffer. It can be described by following set of equations:

$i_{\mathrm{Y}}=0, v_{\mathrm{X}}=i_{\mathrm{X}} R_{\mathrm{X}}+\beta(s) v_{\mathrm{Y}}, i_{\mathrm{Z}}=\alpha(s) i_{\mathrm{X}}, v_{\mathrm{W} \pm}= \pm \mu_{\mathrm{W} \pm}(s) v_{\mathrm{Z}}$,

where $R_{\mathrm{X}}$ is a non-zero intrinsic resistance at input terminal $\mathrm{X}$ and $\alpha(s), \beta(s), \mu_{\mathrm{w}^{+}}(s), \mu_{\mathrm{w}-}(s)$ are respectively the current and voltage gains that can be expressed as $\alpha(s)=\omega_{\alpha}\left(1-\varepsilon_{\alpha}\right) /\left(s+\omega_{\alpha}\right), \quad \beta(s)=\omega_{\beta}\left(1-\varepsilon_{\beta}\right) /\left(s+\omega_{\beta}\right), \quad \mu_{\mathrm{w}+}(s)=$ $\omega_{\mu \mathrm{w}+}\left(1-\varepsilon_{\mu \mathrm{w}+}\right) /\left(s+\omega_{\mu \mathrm{w}+}\right), \quad \mu_{\mathrm{w}-}(s)=\omega_{\mu \mathrm{w}-}\left(1-\varepsilon_{\mu \mathrm{w}-}\right) /\left(s+\omega_{\mu \mathrm{w}-}\right)$ with $\left|\varepsilon_{\alpha}\right| \ll 1,\left|\varepsilon_{\beta}\right| \ll 1,\left|\varepsilon_{\mu_{\mathrm{w}}}\right| \ll 1$, and $\left|\varepsilon_{\mu_{\mathrm{w}}}\right| \ll 1$. Here, the $\varepsilon_{\alpha}$ denotes the current tracking error, $\varepsilon_{\beta}, \varepsilon_{\mu \mathrm{w}+}$, and $\varepsilon_{\mu \mathrm{w}-}$ denote the voltage tracking errors, while $\omega_{\alpha}, \omega_{\beta}, \omega_{\mu \mathrm{w}+}, \omega_{\mu \mathrm{w}^{-}}$denote corresponding corner frequencies. Note that in the ideal case, the current and voltage gains are unity i.e. $\alpha(s)=1, \beta(s)=\mu_{\mathrm{w}+}(s)=\mu_{\mathrm{w}-}(s)=1$ and frequency independent. 


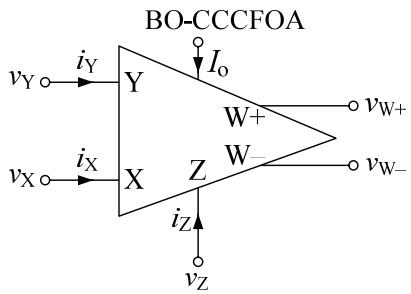

(a)

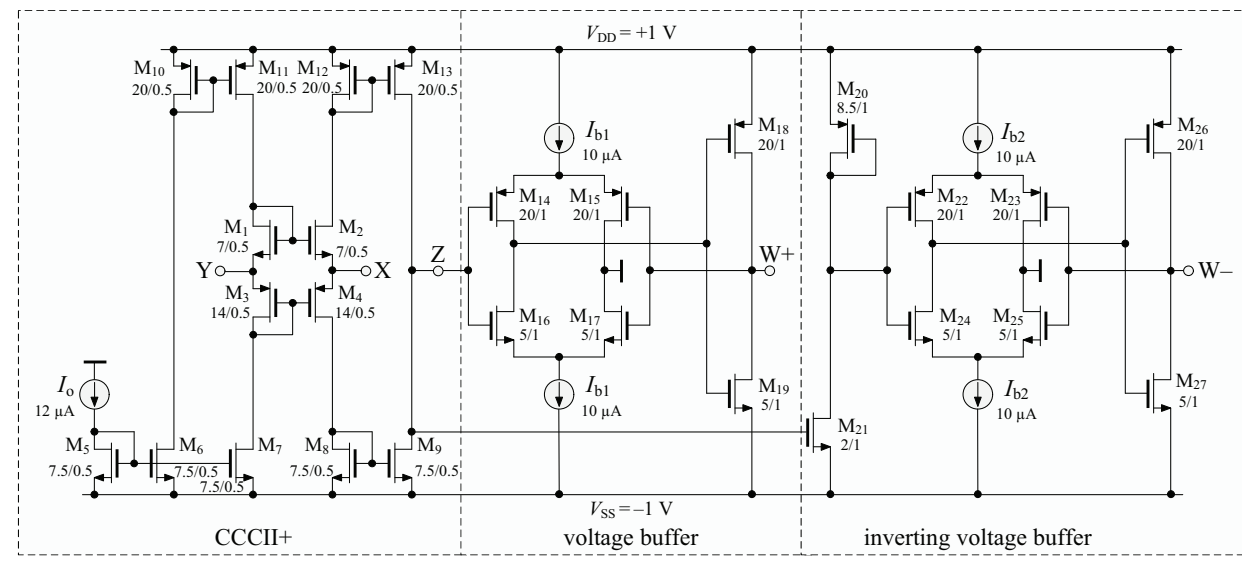

(b)

Fig. 1. (a) Circuit symbol and (b) CMOS implementation of BO-CCCFOA.

The proposed CMOS implementation of the BO-CCCFOA is given in Fig. 1(b), where transistors $M_{1}-M_{13}$ form a CCCII+ and transistors $\mathrm{M}_{14}-\mathrm{M}_{19}$ and $\mathrm{M}_{20}-\mathrm{M}_{27}$ are used for realizing the voltage buffer with balanced outputs. In addition, intrinsic input resistance $R_{\mathrm{X}}$ can be electronically controlled and it can be expressed as $R_{\mathrm{X}}=\left(8 I_{\mathrm{O}} K\right)^{-1 / 2}$. Here, $I_{\mathrm{o}}$ is DC bias current and $K=\mu_{\mathrm{P}} C_{\mathrm{OX}}(W / L)_{3,4}=\mu_{\mathrm{N}} C_{\mathrm{OX}}(W / L)_{1,2}$ is the physical parameter of the corresponding MOS transistors, where $\mu_{\mathrm{P}, \mathrm{N}}$ is electron mobility in the channel, $C_{\mathrm{OX}}$ is the gate-oxide capacitance per unit area, and $W / L$ are transistors effective channel width length, respectively. Considering the available CFOA realizations with electronic tuning, here reported one is extension of [3] in terms of balance voltage outputs, but less complex due to reduction of current gain control stage in [4].

\section{INDUCTANCE SIMULATOR DESIGN}

Symbol and the proposed resistorless lossless grounded PIS employing two ABBs and a single capacitor are shown in Figs. 2(a) and (b), respectively. Assuming an ideal BO-CCCFOAs, i.e. $\alpha_{i}(s)=1$ and $\beta_{i}(s)=\mu_{\mathrm{w}+i}(s)=\mu_{\mathrm{w}-i}(s)=1$ for $i=1$ and 2 , routine circuit analysis yields the following input impedance:

$$
\begin{aligned}
Z_{\text {in } \gamma}(s)=\frac{V_{\text {in }}}{I_{\text {in }}}=s L_{\gamma} & =s C_{\gamma} R_{\mathrm{X} 1} R_{\mathrm{X} 2}= \\
& =\frac{s C_{\gamma}}{8 \sqrt{I_{\mathrm{o} 1} I_{\mathrm{o} 2} K_{1} K_{2}}} .
\end{aligned}
$$

From (2) it is obvious that the equivalent inductance value $L_{\gamma}=C_{\gamma} R_{\mathrm{X} 1} R_{\mathrm{X} 2}$ of the proposed lossless PIS in Fig. 2(b) can be adjusted electronically by either bias currents $\left(I_{\mathrm{o} 1}, I_{\mathrm{o} 2}\right)$ and/or by order of the FoC (or phase). The Foster II structure depicted in Fig. 2(b) has been used to realize the FoC with a fractionalorder of $\gamma=(0.25 ; 0.5 ; 0.75)$.

\section{Simulation Results}

To verify the theoretical analysis, the proposed circuits have been simulated using SPICE program. DC power supply

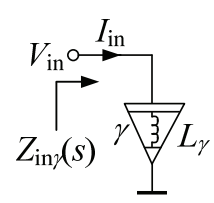

(a)

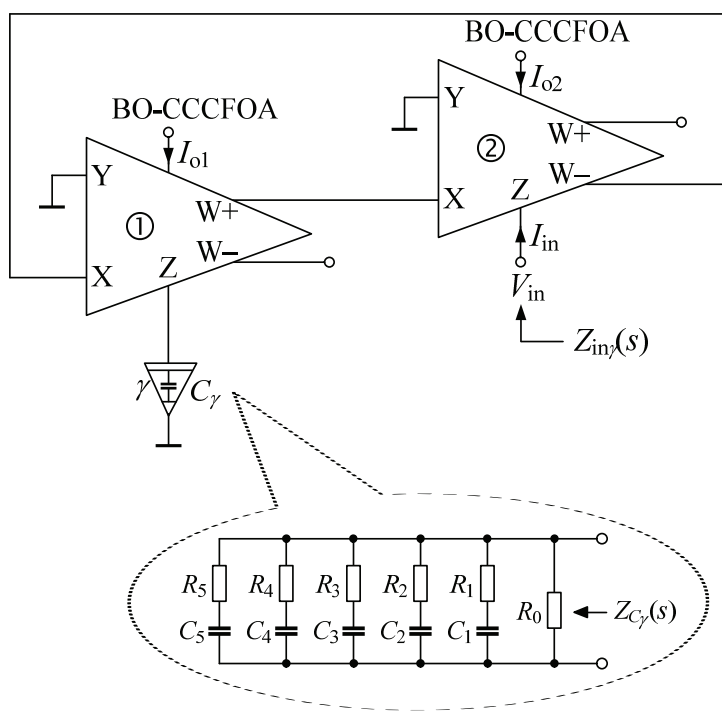

(b)

Fig. 2. (a) Symbol of grounded PIS, (b) proposed FoI simulator including RC network emulating FoC.

voltages of designed CMOS implementation of BO-CCCFOA shown in Fig. 1(b) were set $+V_{\mathrm{DD}}=-V_{\mathrm{SS}}=1 \mathrm{~V}$. In the design, transistors are modeled by the TSMC $0.18 \mu \mathrm{m}$ level-7 LO EPI SCN018 CMOS process parameters $\left(V_{\mathrm{THN}}=0.3725 \mathrm{~V}\right.$, $\mu_{\mathrm{N}}=259.5304 \mathrm{~cm}^{2} /(\mathrm{V} \cdot \mathrm{s}), \quad V_{\mathrm{THP}}=-0.3948 \mathrm{~V}, \quad \mu_{\mathrm{P}}=109.9762$ $\left.\mathrm{cm}^{2} /(\mathrm{V} \cdot \mathrm{s}), T_{\mathrm{OX}}=4.1 \mathrm{~nm}\right)$ [7]. The aspect ratios of CMOS transistors are depicted in the structure. During simulations the bias current of input circuitry was set as $I_{\mathrm{o}}=12 \mu \mathrm{A}$, which resulted in an intrinsic resistance at input $\mathrm{X}$ terminal $R_{\mathrm{X}} \cong 2.4 \mathrm{k} \Omega$, while $I_{\mathrm{b} 1}=I_{\mathrm{b} 2}=10 \mu \mathrm{A}$. 
TABle I. Component Values Used in Fig. 2(B) for SPICE Simulations of C=300 pF @ 407.5 kHz. (Note: ${ }^{*}$ IN 30 kHz - 30 MHz RANGe)

\begin{tabular}{|c|c|c|c|}
\hline $\begin{array}{l}\text { Component } \\
\text { values }\end{array}$ & $\begin{array}{c}\text { Order: } \gamma=0.25 \\
\text { Phase: }-22.5 \text { degree } \\
\text { Pseudo-capacitance: } C_{0.25}=19.2 \mu \mathrm{F} \cdot \sec ^{-0.75}\end{array}$ & $\begin{array}{c}\text { Order: } \gamma=0.5 \\
\text { Phase: }-45 \text { degree } \\
\text { Pseudo-capacitance: } C_{0.5}=480 \mathrm{nF} \cdot \mathrm{sec}^{-0.5}\end{array}$ & $\begin{array}{c}\text { Order: } \gamma=0.75 \\
\text { Phase: }-67.5 \text { degree } \\
\text { Pseudo-capacitance: } C_{0.75}=12 \mathrm{nF} \cdot \mathrm{sec}^{-0.25}\end{array}$ \\
\hline$R_{0}(\mathrm{k} \Omega)$ & 3.48 & 14.7 & 51.1 \\
\hline$R_{1}(\Omega)$ & 619 & 75 & 10 \\
\hline$R_{2}(\Omega)$ & 1620 & 511 & 261 \\
\hline$R_{3}(\mathrm{k} \Omega)$ & 1000 & 1.33 & 0.909 \\
\hline$R_{4}(\mathrm{k} \Omega)$ & 2.87 & 3.16 & 3.48 \\
\hline$R_{5}(\mathrm{k} \Omega)$ & 3.83 & 6.81 & 13.3 \\
\hline$C_{1}(\mathrm{pF})$ & 7.5 & 27 & 91 \\
\hline$C_{2}(\mathrm{pF})$ & 27 & 43 & 51 \\
\hline$C_{3}(\mathrm{pF})$ & 2200 & 100 & 82 \\
\hline$C_{4}(\mathrm{pF})$ & 120 & 240 & 130 \\
\hline \multirow[t]{9}{*}{$C_{5}(\mathrm{pF})$} & 750 & 750 & 240 \\
\hline & \multicolumn{3}{|c|}{ Total resistance $(\mathrm{k} \Omega)$ / capacitance $(\mathrm{nF})$} \\
\hline & $1012.42 / 3.11$ & $26.59 / 1.16$ & $69.06 / 0.594$ \\
\hline & \multicolumn{3}{|c|}{ Spread of resistance / capacitance } \\
\hline & $1615.51 / 293.33$ & $196 / 27.78$ & $5110 / 4.71$ \\
\hline & \multicolumn{3}{|c|}{ Mean values for phase angle deviation ${ }^{\#}($ degree $) /$ relative phase $\operatorname{error}^{\#}(\%)$} \\
\hline & $\pm 0.95 / \pm 4.24$ & $\pm 0.81 / \pm 1.81$ & $\pm 0.66 / \pm 0.97$ \\
\hline & \multicolumn{3}{|c|}{ Equations for fitting the phase and pseudo-capacitance via linear regression ${ }^{\#}$} \\
\hline & $\begin{array}{l}y_{\text {Phase }}=-1.78 \cdot 10^{-8} \cdot x-22.52 \\
y_{C \gamma}=3.35 \cdot 10^{-14} \cdot x+1.9 \cdot 10^{-5}\end{array}$ & $\begin{array}{c}y_{\text {Phase }}=3.9 \cdot 10^{-9} \cdot x-45.09 \\
y_{C \gamma}=2.12 \cdot 10^{-16} \cdot x+4.85 \cdot 10^{-7}\end{array}$ & $\begin{array}{c}y_{\text {Phase }}=1.67 \cdot 10^{-8} \cdot x-67.22 \\
y_{C \gamma}=-2.83 \cdot 10^{-18} \cdot x+1.21 \cdot 10^{-8}\end{array}$ \\
\hline
\end{tabular}

In order to verify the workability of the proposed grounded lossless PIS circuit with various orders, a $5^{\text {th }}$-order Foster II RC network shown in Fig. 2(b) was used with orders $\gamma=(0.25 ; 0.5$; 0.75), which has an admittance in a form as follows $Y_{C_{\gamma}}=1 / Z_{C_{\gamma}}=1 / R_{0}+\sum_{k=1}^{5} s C_{k} /\left[s R_{k} C_{k}+1\right]$. Note that the component values optimized for constant phase in $30 \mathrm{kHz}$ $30 \mathrm{MHz}$ frequency range with phase angle deviation below \pm 1 degree using MLSQ method are listed in Table I. The total resistance/capacitance and spread of resistance/capacitance for each FoC realization is investigated. In addition, the equivalent equations for fitting the phase and pseudo-capacitance via linear regression are also provided. Simulated phase and (pseudo)-inductance responses of $\gamma=(0.25 ; 0.5 ; 0.75 ; 1)$-order inductance simulator are shown in Fig. 3. Due to the BOCCCFOA non-idealities, the performance of the proposed FoI is restricted. Hence, considering the bandwidth for phase angle deviation up to 3 degree, the useful frequency ranges for designed equivalent $L_{\gamma}=\left(110.32 \mathrm{H} \cdot \mathrm{s}^{-0.75} ; \quad 2.76 \mathrm{H} \cdot \mathrm{s}^{-0.5}\right.$; $\left.68.95 \mathrm{mH} \cdot \mathrm{s}^{-0.25} ; 1.72 \mathrm{mH}\right)$ are listed in Table II. Moreover, Fig. 4 shows the effect of $C_{\gamma}$ vs. $\gamma$ on FoI magnitude.

To demonstrate the usefulness of the proposed grounded lossless PIS with various orders, it was used in a high-pass filter (HPF) realization. The passive $\mathrm{RL}_{\gamma} \mathrm{C}$ prototype is shown in Fig. 5 and its voltage transfer function is given by:

$$
T_{\mathrm{VM}}(s)=\frac{V_{\text {out }}}{V_{\text {in }}}=\frac{s^{2+\gamma} C_{\mathrm{s} 1} C_{\mathrm{s} 2} L_{\gamma} R_{\mathrm{p}}}{s^{2+\gamma} a_{4}+s^{2} a_{3}+s^{1+\gamma} a_{2}+s a_{1}+a_{0}} .
$$

TABLE II. PERFORMANCE COMPARISON OF FOI IMPLEMENTATION.

\begin{tabular}{|c|c|c|c|c|}
\hline \multirow{2}{*}{$\begin{array}{c}\text { Component } \\
\text { values }\end{array}$} & \multicolumn{4}{|c|}{ Order / Phase } \\
\cline { 2 - 5 } & $\begin{array}{c}\gamma=\mathbf{0 . 2 5} \\
\mathbf{2 2 . 5} \text { degree }\end{array}$ & $\begin{array}{c}\gamma=\mathbf{0 . 5} \\
\mathbf{4 5} \text { degree }\end{array}$ & $\begin{array}{c}\gamma=\mathbf{0 . 7 5} \\
\mathbf{6 7 . 5} \text { degree }\end{array}$ & $\begin{array}{c}\gamma=\mathbf{1} \\
\mathbf{9 0} \text { degree }\end{array}$ \\
\hline $\begin{array}{c}\text { Bandwidth for } \\
\text { phase angle } \\
\text { deviation }\end{array}$ & $\begin{array}{c}24.4 \mathrm{kHz} \\
\downarrow\end{array}$ & $\begin{array}{c}13.2 \mathrm{kHz} \\
\downarrow\end{array}$ & $\begin{array}{c}24.6 \mathrm{kHz} \\
\downarrow\end{array}$ & $\begin{array}{c}44.2 \mathrm{kHz} \\
\downarrow\end{array}$ \\
$<3$ degree & $2 \mathrm{MHz}$ & $3.45 \mathrm{MHz}$ & $20.5 \mathrm{MHz}$ & $3.43 \mathrm{MHz}$ \\
\hline
\end{tabular}

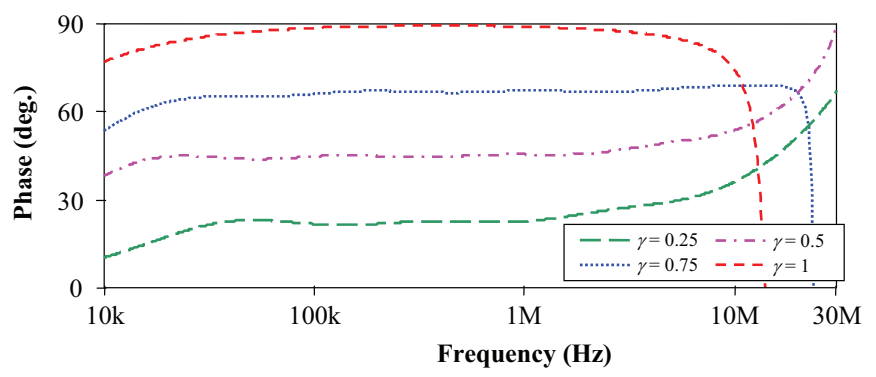

(a)

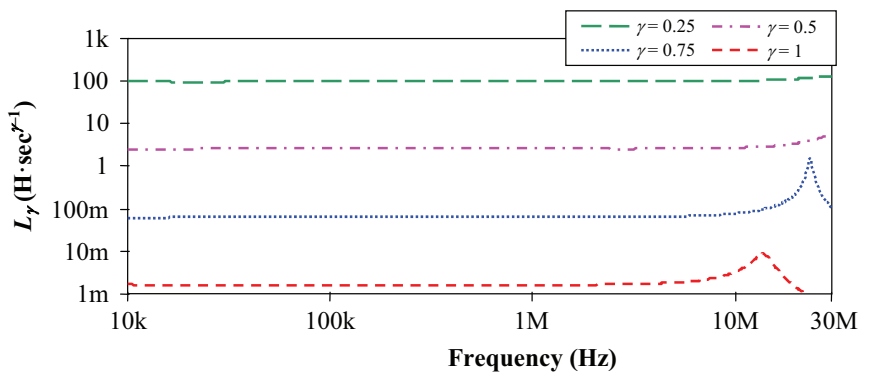

(b)

Fig. 3. Simulated (a) phase and (b) (pseudo)-inductance responses of FoI. 
TABLE III. COMPONENT VALUES USED IN HPF RL $\gamma$ C PROTOTYPE.

\begin{tabular}{|c|c|c|c|c|}
\hline \multirow{2}{*}{$\begin{array}{c}\text { Component } \\
\text { values }\end{array}$} & \multicolumn{4}{|c|}{ Order / Phase } \\
\cline { 2 - 5 } & $\begin{array}{c}\boldsymbol{\gamma}=\mathbf{0 . 2 5} \\
\mathbf{2 2 . 5} \text { degree }\end{array}$ & $\begin{array}{c}\gamma=\mathbf{0 . 5} \\
\mathbf{4 5} \text { degree }\end{array}$ & $\begin{array}{c}\gamma=\mathbf{0 . 7 5} \\
\mathbf{6 7 . 5} \text { degree }\end{array}$ & $\begin{array}{c}\gamma=\mathbf{1} \\
\mathbf{9 0} \text { degree }\end{array}$ \\
\hline$R_{\mathrm{s}}, R_{\mathrm{p}}(\mathrm{k} \Omega)$ & \multicolumn{4}{|c|}{21.7} \\
\hline$C_{\mathrm{s} 1}, C_{\mathrm{s} 2}(\mathrm{pF})$ & \multicolumn{4}{|c|}{7.334} \\
\hline$L_{\gamma}$ & $110.32 \mathrm{H} \cdot \mathrm{s}^{-0.75}$ & $2.76 \mathrm{H} \cdot \mathrm{s}^{-0.5}$ & $68.95 \mathrm{mH} \cdot \mathrm{s}^{-0.25}$ & $1.72 \mathrm{mH}$ \\
\hline
\end{tabular}

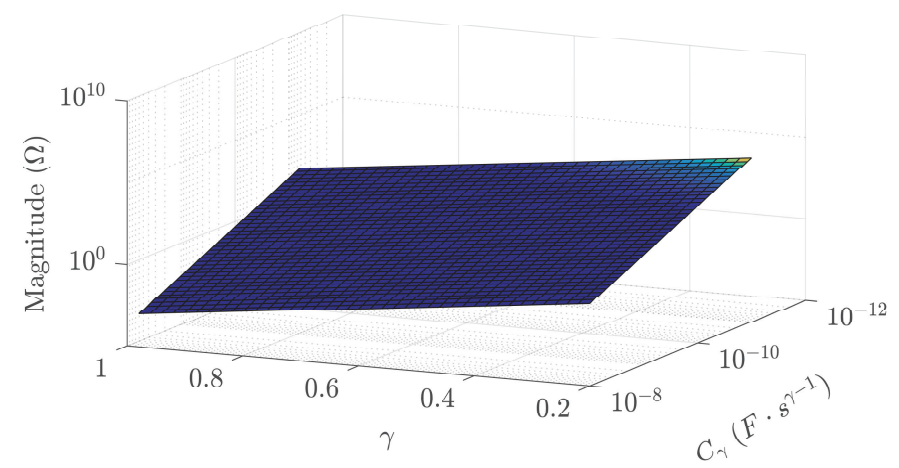

Fig. 4. Effect of $C_{\gamma}$ vs. $\gamma$ on FoI magnitude.

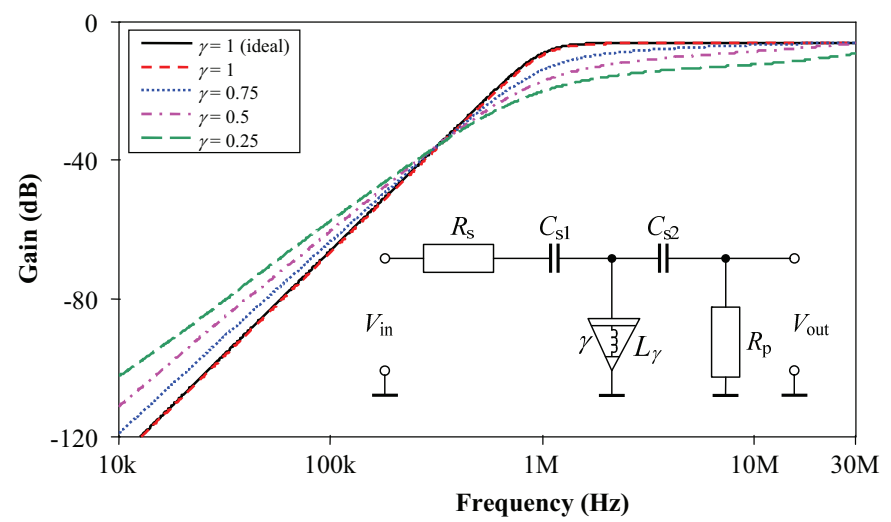

Fig. 5. VM high-pass filter $\mathrm{RL}_{\gamma} \mathrm{C}$ ladder prototype and its ideal and simulated gain characteristics.

In (3), $a_{0}=1, a_{1}=\left(C_{\mathrm{s} 1} R_{\mathrm{s}}+C_{\mathrm{s} 2} R_{\mathrm{p}}\right), a_{2}=L_{\gamma}\left(C_{\mathrm{s} 1}+C_{\mathrm{s} 2}\right)$, $a_{3}=C_{\mathrm{s} 1} C_{\mathrm{s} 2} R_{\mathrm{s}} R_{\mathrm{p}}, \quad a_{4}=C_{\mathrm{s} 1} C_{\mathrm{s} 2} L_{\gamma}\left(R_{\mathrm{s}}+R_{\mathrm{p}}\right) \quad$ and computed passive component values used in HPF $R L_{\gamma} C$ prototype obtained for $1 \mathrm{MHz}$ cut-off frequency with Butterworth approximation (integer-order case) are given in Table III. Simulated gain characteristics of designed VM high-pass filter considering various order $\mathrm{FoI}$ in $\mathrm{RL}_{\gamma} \mathrm{C}$ ladder prototype are again shown in Fig. 5.

\section{BRIEF DISCUSSION}

This section addresses brief discussion on obtained results. First of all, it is worth noting that the phase responses of each demonstrated $\mathrm{FoC}$ in Table II is constant over three decades (in $30 \mathrm{kHz}-30 \mathrm{MHz}$ frequency range) and obtained phase angle deviation is below \pm 1 degree. Subsequently, mean values of corresponding relative phase error in same frequency range is below $\pm 4.3 \%$ for each FoC. Hence, MLSQ method is useful tool for RC networks optimization. On the other hand, the performance of the proposed $\mathrm{FoI}$ is restricted due to nonidealities of the ABB used. However, the proposed FoI still operates over two decades, while its pseudo-inductance varies from $110.32 \mathrm{H} \cdot \mathrm{s}^{-0.75}$ to $68.95 \mathrm{mH} \cdot \mathrm{s}^{-0.25}$ for $\gamma \in[0.25 ; 0.75]$. The effect of $C_{\gamma}$ vs. $\gamma$ on FoI magnitude is demonstrated in Fig. 4. Finally, from obtained results it can be seen that proposed FoC and FoI operate in wide frequency range and they are in very good agreement with the theory.

\section{CONCLUSION}

The paper presented an active building blocked called current-controlled current feedback operational amplifier with balanced voltage outputs and its utilization in grounded lossless positive inductance simulator circuit with various orders. Three broadband FoCs with phase angle deviation below \pm 1 degree were verified. Subsequently, the workability of FoI employing two BO-CCCFOAs and FoCs with various orders were demonstrated in voltage-mode high-pass filter $\mathrm{RL}_{\gamma} \mathrm{C}$ ladder prototype. The theory and the behavior of proposed circuits were verified using SPICE simulations.

\section{REFERENCES}

[1] F. Yuan, CMOS Active Inductors and Transformers: Principle, Implementation, and Applications. Springer Sci. \& Busin. Media, 2008.

[2] R. Senani, D. R. Bhaskar, A. K. Singh, and V. K. Singh, Current feedback operational amplifiers and their applications, Springer, 2013.

[3] M. Siripruchyanun, C. Chanapromma, P. Silapan, and W. Jaikla, "BiCMOS current-controlled current feedback amplifier (CC-CFA) and its applications," WSEAS Trans. on Electron., vol. 5, pp. 203-219, 2008.

[4] R. Sotner, N. Herencsar, J. Jerabek, R. Dvorak, A. Kartci, T. Dostal, and K. Vrba, "New double current controlled CFA (DCC-CFA) based voltage-mode oscillator with independent electronic control of oscillation condition and frequency," Journal of Electrical Engineering, vol. 64, no. 2, pp. 65-75, 2013.

[5] A. Agambayev, S. Patole, H. Bagci, and K. N. Salama, "Tunable fractional-order capacitor using layered ferroelectric polymers," AIP Advances, vol. 7, no. 9, pp. 095202-1-095202-8, 2017.

[6] A. Kartci, A. Agambayev, N. Herencsar, and K.N. Salama, "Series-, parallel-, and inter-connection of solid-state arbitrary fractional-order capacitors: theoretical study and experimental verification," IEEE Access, vol. 6, pp. 10933-10943, 2018.

[7] N. Herencsar, R. Sotner, A. Kartci, and K. Vrba, "A novel pseudodifferential integer/fractional-order voltage-mode all-pass filter," In Prof. of the 2018 IEEE International Symposium on Circuits and Systems (ISCAS), Florence, Italy, 2018, pp. 1-5.

[8] G. Tsirimokou, A. Kartci, J. Koton, N. Herencsar, and C. Psychalinos, "Comparative study of discrete component realizations of fractionalorder capacitor and inductor active emulators," Journal of Circuits, Systems and Computers, vol. 27, no. 11, pp. 1850170, 2018.

[9] A. G. Radwan and K. N. Salama, "Passive and active elements using fractional $\mathrm{L}_{\beta} \mathrm{C}_{\alpha}$ circuit," IEEE Trans. on Circuits and Systems I, vol. 58, no. 10, pp. 2388-2397, 2011.

[10] A. Adhikary, S. Choudhary, and S. Sen, "Optimal design for realizing a grounded fractional order inductor using GIC," IEEE Trans. on Circuits and Systems I, vol. 65, no. 8, pp. 2411-2421, 2018.

[11] R. Sotner, J. Jerabek, N. Herencsar, L. Langhammer, J. Petrzela, and T. Dostal, "Methods for Extension of Tunability Range in Synthetic Inductance Simulators," Elektronika ir Elektrotechnika, vol. 24, no. 3, pp. 41-45, 2018.

[12] J. Koton, D. Kubanek, N. Herencsar, J. Dvorak, and C. Psychalinos, "Designing constant phase elements of complement order," Analog Integrated Circuits and Signal Processing, pp 1-8, 2018. DOI: 10.1007/s10470-018-1257-7 